\title{
New exactly solvable relativistic model with anomalous interaction
}

\author{
Elena Ferrard* and Antonino Messinat \\ INFM, MIUR and \\ Dipartimento di Scienze Fisiche ed Astronomiche dell'Università di Palermo \\ Via Archirafi, 36 - I-90123 Palermo. Italy \\ A.G. Nikitin $\ddagger$ \\ Institute of Mathematics, National Academy of Sciences of Ukraine, \\ 3 Tereshchenkivs'ka Street, Kyiv-4, Ukraine, 01601
}

(Dated: November 24, 2018)

\begin{abstract}
A special class of Dirac-Pauli equations with time-like vector potentials of external field is investigated. A new exactly solvable relativistic model describing anomalous interaction of a neutral Dirac fermion with a cylindrically symmetric external e.m. field is presented. The related external field is a superposition of the electric field generated by a charged infinite filament and the magnetic field generated by a straight line current. In non-relativistic approximation the considered model is reduced to the integrable Pron'ko-Stroganov model.
\end{abstract}

PACS numbers: 03.65.Pm,03.65.Fd, 03.65.Ge, 11.30.Pb

*Electronic address: ferraro@fisica.unipa.it

${ }^{\dagger}$ Electronic address: messina@fisica.unipa.it

${ }^{\ddagger}$ Electronic address: nikitin@imath.kiev.ua 


\section{INTRODUCTION}

Exact solutions of relativistic wave equations are both very rare and important. First they provide explicit solutions to concrete physical problems free of inaccuracies and inconveniences of approximate methods. Secondly, such exact solutions can serve as convenient basis sets for expanding the solutions of other physical problems which are not necessarily exactly solvable.

A good survey of exact solutions of relativistic wave equations can be found in [1]. Notwithstanding this book has been published as long ago as in 1990, it continues to be a good information resource on exactly solvable relativistic systems for particles with spins 0 and 1/2. Surely this collection is not exhausting: many new results have been obtained during two latest decades, including the problems for the Dirac equation in lower dimension space and the problems for neutral Dirac particles.

Exact solutions of the Dirac equation describing electrically neutral particles with nonminimal interaction with an external electromagnetic field are noteworthy. Physically, such solutions have the very big application value since they can be used to model the motion of a neutron in realistic situations. In particular they have relations to the nuclear reactors security problems. Moreover, magnetic trapping of neutrons is a subject of direct experimental studies, refer, e.g., to [2]. Mathematically, the anomalous interaction terms depending on tensor fields dramatically reduce the number of problems which can be solved using complete separation of variables. In addition, just neutral particles anomalously coupled to the external magnetic field give rise of the Aharonov-Casher effect [3] with its interesting physical and mathematical aspects.

The very possibility to solve a problem exactly stems from the existence of a dynamical symmetry which is more extended than the geometric symmetry of the problem. The famous examples of such exactly solvable systems are the Kepler problems and isotropic oscillator whose dynamical symmetries are defined by groups $\mathrm{SO}(4)$ and $\mathrm{U}(3)$ respectively. One more well known example is the Pron'ko-Stroganov (PS) problem [4] which describes the anomalous interaction of a non-relativistic electrically neutral particle of spin $1 / 2$ with the field of a straight line current. The related dynamical symmetry of negative energy states is described by group $\mathrm{SO}(3)$ while the geometrical symmetry of the system is reduced to the rotation group in two dimensions, i.e., to $\mathrm{SO}(2)$. Let us stress that the PS problem was formulated for the Schrödinger-Pauli equation for neutral particles, i.e., it is essentially nonrelativistic. 
Paper [4] was followed by a number of publications devoted to exactly solvable problems for neutral particles. In particular, the supersymmetric aspects of the PS model were investigated in [5]-[7], more realistic models based on magnetic field produced by current of thin filament were discussed in [8] and [6] following the non-relativistic approach. A rather completed study of the Dirac-Pauli equation for neutral particles can be found in [9], the case of purely electric time-independent external field was studied in [10]. However, an exactly solvable relativistic analogue of the PS problem was not known till now.

We can add that searching for exact solutions of Dirac equation belongs to evergreen problems, apparently the most recent result in this field can be found in [11]. Exactly solvable two-particle Dirac equations are discussed, e.g., in [12]. For exact solutions of relativistic wave equations for particles with higher and arbitrary spins see [13] and [14].

In the present paper we discuss a certain class of relativistic problems describing the anomalous interaction of the Dirac fermion with an external electromagnetic field. The considered equations admit an effective reduction to equations invariant with respect to the $1+2$ dimensional Galilei group, which can be made by using the light cone coordinates.

Let us remind that the light cone coordinates was introduced by Dirac [15] in an attempt to formulate a relativistic dynamics with the direct interaction. Then it was recognized that these coordinates present powerful tools for solution of relativistic wave equations which include plane wave potentials or, more generally, if the potentials do not depend on a scalar products of the coordinate vector with a constant time-like vector [16], [17].

We show that the considered class of equations includes new exactly solvable relativistic systems and we study in detail one of them, namely the one closely related to both the Pron'koStroganov problem and relativistic problems for the neutron interacting with an external field. The corresponding external field is a superposition of the magnetic field generated by the straight line constant current and the electric field of a charged infinite filament. In spite of that our main goal is to present an exactly solvable problem for neutral fermions, for the sake of generality we consider also more general problems with both the minimal and anomalous interactions. We also indicate an exactly solvable model of this general type, see Section 7 . 


\section{DIRAC-PAULI EQUATIONS AND REDUCTION SO $(1,3) \rightarrow \mathrm{HG}(1,2)$}

Consider the Dirac-Pauli equation for a charged particle which interacts anomalously with an external electromagnetic field:

$$
\left(\gamma^{\mu} \pi_{\mu}-m-\lambda S^{\mu \nu} F_{\mu \nu}\right) \psi=0
$$

Here $\pi_{\mu}=p_{\mu}-e A_{\mu}, p_{\mu}=\mathrm{i} \frac{\partial}{\partial x^{\mu}}, A_{\mu}$ are components of the vector-potential of the external electromagnetic field, $\gamma^{\mu}$ are Dirac matrices satisfying the Clifford algebra

$$
\gamma^{\mu} \gamma^{\nu}+\gamma^{\nu} \gamma^{\mu}=2 g^{\mu \nu}
$$

$g^{\mu \nu}$ is the metric tensor whose nonzero elements are $g^{00}=-g^{11}=-g^{22}=-g^{33}=1, S^{\mu \nu}=$ $\mathrm{i}\left(\gamma^{\mu} \gamma^{\nu}-\gamma^{\nu} \gamma^{\mu}\right) / 4$ is the spin tensor, $F_{\mu \nu}=\mathrm{i}\left[\pi_{\mu}, \pi_{\nu}\right]$ is the tensor of the electromagnetic field such that $F_{0 a}=-E_{a}, F_{a b}=\varepsilon_{a b c} B_{c}$ where $E_{a}$ and $B_{c}$ are components of vectors of the electric and magnetic field strengthes. In addition, $e$ and $\lambda$ denote particle charge and constant of anomalous coupling. The latest is usually represented as

$$
\lambda=g \mu_{0}
$$

where $\mu_{0}$ is the Bohr magneton and $g$ is the Landé factor.

We use Heaviside units with $\hbar=c=1$.

Equation (2.1) describes both the minimal and anomalous interaction of the Dirac fermion with an external electromagnetic field. Setting in (2.1) $\lambda=0$ and supposing $e \neq 0$ we come to the equation describing the anomalous interaction only, while for $e=0, \lambda \neq 0$ we obtain the Dirac-Pauli equation describing a neutral fermion. In the latest case the parameter $g$ in (2.3) is just the contribution to the Landé factor arising from the anomalous magnetic moment.

Equation (2.1) is transparently invariant with respect to the Lorentz group $\mathrm{SO}(1,3)$ which transforms time and space variables $x_{0}, x_{1}, x_{2}, x_{3}$ between themselves. Among the subgroups of this group there is the homogeneous Galilei group $\operatorname{HG}(1,2)$ which includes the transformations of variables $\tau, x_{1}, x_{2}$ where $\tau=\left(x_{0}+x_{3}\right) / 2$ (for all non-equivalent subgroups of $\mathrm{SO}(1,3)$ and of the Poincaré group see [18]).

To search for exactly solvable problems based on equations (2.1) we restrict ourselves to special class of external fields, which makes it possible to expand solutions of (2.1) via solutions of reduced equations invariant w.r.t. group $\mathrm{HG}(1,2)$. In other words, we will discuss such external fields for which these reduced equations be integrable. 
To this end we first suppose that the vector-potential $A=\left(A_{0}, A_{1}, A_{2}, A_{3}\right)$ be light-like, i.e.,

$$
A_{\mu} A^{\mu}=0 .
$$

This condition can be always satisfied up to gauge transformations and so it does not lead to any loss of generality. Then we restrict ourselves to the vector-potentials of the following special form compatible with (2.4):

$$
A=(\varphi, 0,0, \varphi)
$$

where $\varphi$ is a function of time and spatial variables. In addition we suppose that $\varphi$ depends on three variables only, namely,

$$
\varphi=\varphi\left(\tau, x_{1}, x_{2}\right) .
$$

Vector-potentials (2.5), (2.6) satisfy the Lorentz gauge condition $p_{\mu} A^{\mu}=0$ identically and the invariants of the related external field are both equal to zero, i.e.,

$$
F_{\mu \nu} F^{\mu \nu}=0 \text { and } \frac{1}{2} \varepsilon_{\mu \nu \rho \sigma} F^{\mu \nu} F^{\rho \sigma}=0 .
$$

For convenience we fix a nonstandard realization of the Dirac matrices and set

$$
\gamma^{0}=\left(\begin{array}{ll}
0 & I \\
I & 0
\end{array}\right), \gamma^{3}=\left(\begin{array}{rr}
0 & I \\
-I & 0
\end{array}\right), \quad \gamma^{\alpha}=\mathrm{i}\left(\begin{array}{cc}
\sigma_{\alpha} & 0 \\
\mathbf{0} & -\sigma_{\alpha}
\end{array}\right)
$$

where $\alpha=1,2, \quad \sigma_{\alpha}$ are Pauli matrices, $\mathbf{0}$ and $I$ are the $2 \times 2$ zero and unit matrix correspondingly. Then

$$
S^{0 \alpha}=\frac{1}{2}\left(\begin{array}{cc}
\mathbf{0} & \sigma_{\alpha} \\
-\sigma_{\alpha} & \mathbf{0}
\end{array}\right), \quad S^{3 \alpha}=\frac{1}{2}\left(\begin{array}{cc}
\mathbf{0} & \sigma_{\alpha} \\
\sigma_{\alpha} & \mathbf{0}
\end{array}\right) .
$$

System (2.1) with a particular class of the vector-potential given by equations (2.5) and (2.6) is homogeneous with respect to the sum of independent variables $x_{0}+x_{3}$. Thus it is convenient to rewrite it in the light cone variables

$$
\tau=\frac{1}{2}\left(x_{0}-x_{3}\right) \text { and } \xi=\frac{1}{2}\left(x_{0}+x_{3}\right) .
$$

As a result we obtain:

$$
L \psi \equiv\left(\tilde{\gamma}_{\mu} \tilde{\pi}^{\mu}-m-\lambda \eta_{\alpha} F_{\alpha}\right) \psi=0
$$


where $F_{\alpha}=\frac{\partial \varphi}{\partial x_{\alpha}}, \alpha=1,2$,

$$
\begin{gathered}
\tilde{\gamma}_{0}=\gamma_{0}+\gamma_{3}, \tilde{\gamma}_{3}=\frac{1}{2}\left(\gamma_{0}-\gamma_{3}\right), \tilde{\gamma}_{\alpha}=\gamma_{\alpha} \\
\eta_{\alpha}=\frac{1}{2}\left(\gamma_{0} \gamma_{\alpha}+\gamma_{3} \gamma_{\alpha}\right), \tilde{\pi}_{0}=\mathrm{i} \frac{\partial}{\partial \tau}-2 e \varphi \\
\tilde{\pi}_{3}=2 P_{3}=2 \mathrm{i} \frac{\partial}{\partial \xi}, \tilde{\pi}_{\alpha}=p_{\alpha}=-\mathrm{i} \frac{\partial}{\partial x_{\alpha}}
\end{gathered}
$$

and summation w.r.t. repeated indices $\mu$ and $\alpha$ is imposed over the values $\mu=0,1,2,3$ and $\alpha=1,2$ respectively. In addition, we impose on solutions of (2.10) the standard condition of square integrability and ask for $\psi \rightarrow 0$ when $x_{\alpha} \rightarrow 0$.

Operator $P_{3}$ commutes with $L$ and so is a constant of the motion for equation (2.10). Let us expand solutions of this equation via eigenvectors $\psi_{M}$ of $P_{3}$ :

$$
P_{3} \psi_{M}=M \psi_{M} \Rightarrow \psi_{M}=\exp (\mathrm{i} M \xi) \psi\left(\tau, x_{1}, x_{2}\right)
$$

Let us denote

$$
\psi\left(\tau, x_{1}, x_{2}\right)=\left(\begin{array}{c}
\rho\left(\tau, x_{1}, x_{2}\right) \\
\chi\left(\tau, x_{1}, x_{2}\right)
\end{array}\right)
$$

where $\rho$ and $\chi$ are two-component spinors. Substituting (2.12) and (2.13) into (2.10) and using realization (2.8) of $\gamma$-matrices we obtain the following system:

$$
\begin{gathered}
\left(\mathrm{i} \sigma_{\alpha} p_{\alpha}-m\right) \rho+\left(\mathrm{i} \frac{\partial}{\partial \tau}-2 e \varphi-\lambda \sigma_{\alpha} F_{\alpha}\right) \chi=0 \\
2 M \rho-\left(\mathrm{i} \sigma_{\alpha} p_{\alpha}+m\right) \chi=0 .
\end{gathered}
$$

It is easy to convince oneself from eqs.(2.14), (2.15) that, without loss of generality we can assume that $M$ cannot take the zero value. Indeed, setting $M=0$ in (2.15) we reduce it to the equation for $\chi$ which does not have non-trivial normalizable solutions. Then, equating $\chi$ to zero in (2.14), we obtain the equation for $\rho$ whose normalizable solutions are trivial also.

It is interesting to note that this system is nothing but a $(1+2)$-dimensional version of the Galilei-invariant Lévi-Leblond equation [19] with anomalous interaction, as can be immediately deduced by comparing (2.14) and (2.15) with equation (52) for $e=k=0$ in [20]. Solving eq.(2.15) for $\rho$ under the condition $M \neq 0$ and substituting it into eq.(2.14) we obtain the Schrödinger-Pauli equation for the two-component spinor $\chi$ :

$$
\mathrm{i} \frac{\partial \chi}{\partial \tau}=\left(\varepsilon_{0}+\frac{p^{2}}{2 M}+2 e \varphi+\lambda \sigma_{\alpha} F_{\alpha}\right) \chi
$$


where $\varepsilon_{0}=\frac{m^{2}}{2 M}$ and $p^{2}=p_{1}^{2}+p_{2}^{2}$.

Surely equations (2.16) are more easy to handle then the initial equation (2.1), since they include smaller numbers of dependent and independent variables. In particular, a number of exactly (and quasi-exactly) solvable Scrödinger-Pauli equations (2.16) is well studied, and many of them can be used to construct solvable relativistic problems using the scheme inverse to the previously proposed.

In Section 4 we use this idea to generate a relativistic analogue of the PS problem.

\section{CYLINDRICALLY SYMMETRIC POTENTIALS}

Consider in more details a physically interesting subclass of equations (2.1), (2.5), (2.6) when the corresponding potential $\varphi$ depends on the square $x^{2}=x_{1}^{2}+x_{2}^{2}$ of 2 -vector $\mathbf{x}=\left(x_{1}, x_{2}\right)$ and is independent on $\tau$. The related reduced equation (2.16) takes the form

$$
\mathrm{i} \frac{\partial \chi}{\partial \tau}=H \chi
$$

where

$$
H=\varepsilon_{0}+\frac{p^{2}}{2 M}+2 e \varphi+\lambda \frac{\sigma_{\alpha} x_{\alpha}}{x} \frac{\partial \varphi}{\partial x} .
$$

Equation (3.17) has three additional constants of motion, namely,

$$
P_{0}=\mathrm{i} \frac{\partial}{\partial \tau}, J_{12}=x_{1} p_{2}-x_{2} p_{1}+\frac{\mathrm{i}}{2} \sigma_{3}, \quad Q=\sigma_{1} R_{1}
$$

where $R_{1}$ is the reflection operator which acts on $\chi$ as follows:

$$
R_{1} \chi\left(\tau, x_{1}, x_{2}\right)=\chi\left(\tau,-x_{1}, x_{2}\right)
$$

Operators $P_{0}$ and $J_{12}$ are generator of shifts w.r.t. variable $\tau$ and rotation generator correspondingly. They commute with the Hamiltonian (3.18) and between themselves. Expanding solutions of (3.17) via complete sets of eigenfunctions of $P_{0}$ and $J_{12}$ it is possible to separate variables in this equation.

Operator $Q$ represents a discrete symmetry w.r.t. the reflection of the first coordinate axis. It commutes with $P_{0}$ and $H(\underline{3.18})$ but anticommutes with $J_{12}$. It follows from the above that eigenvalues of $P_{0}$ and $H$ should be degenerated w.r.t. the sign of eigenvalues of $J_{12}$.

Notice that equation (3.17) admits other discrete symmetries like reflections of $x_{2}$ or both $x_{1}$ and $x_{2}$. But all such additional symmetries are either rotation transformations or products of reflection $Q$ and rotations. 
Let us separate variables in equation (3.17). First we define the eigenvectors of $P_{0}$ which have the following form:

$$
\chi_{\varepsilon}=\exp (-\mathrm{i} \varepsilon \tau) \chi(\mathbf{x})
$$

Then, substituting eq.(3.20) into eq.(3.17) we obtain the equation

$$
\varepsilon \chi=H \chi
$$

where $H$ is Hamiltonian (3.18).

In addition to the coupling constants $e$ and $\lambda$, equation(3.21) includes two parameters, i.e., $\varepsilon$ and $M$. For a fixed non-zero $M$ this equation defines an eigenvalue problem for $\varepsilon$.

Now we can use the symmetry of (3.21) w.r.t. the rotation group (whose generator is $J_{12}$ ) to separate radial and angular variables. To do this we rewrite equation (3.21) in terms of angular variables, i.e., set $x_{1}=x \cos \theta, x_{2}=x \sin \theta, r=2 M|\tilde{\lambda}| x$ (where $\tilde{\lambda}$ is a normalizing parameter), and expand $\chi$ via eigenfunctions of the angular momentum operator $J_{12}$ :

$$
\chi=C_{k} \chi_{k}, \quad \chi_{k}=\frac{1}{\sqrt{r}}\left(\begin{array}{c}
\exp \left(\mathrm{i}\left(k-\frac{1}{2}\right) \theta\right) \phi_{1} \\
\epsilon \exp \left(\mathrm{i}\left(k+\frac{1}{2}\right) \theta\right) \phi_{2}
\end{array}\right)
$$

where $C_{k}$ are constants, $\epsilon=\tilde{\lambda} /|\tilde{\lambda}|, \quad \phi_{1}$ and $\phi_{2}$ are functions of $r$ and summation is imposed over the repeated indices $k= \pm \frac{1}{2}, \pm \frac{3}{2}, \pm \frac{5}{2} \cdots$.

In the following we restrict ourselves to solutions $\chi_{k}$ which correspond to non-negative values of $k$. Then solutions with $k$ negative would be obtained by acting on $\chi_{k}$ by operator $Q$ (3.19) .

Substituting eq.(3.22) into eq.(3.21) we come to the following system:

$$
H_{k} \phi \equiv\left(-\frac{\partial^{2}}{\partial r^{2}}+k\left(k-\sigma_{3}\right) \frac{1}{r^{2}}+2 e \varphi+\sigma_{1} \frac{\lambda}{\tilde{\lambda}} \frac{\partial \varphi}{\partial r}\right) \phi=\tilde{\varepsilon} \phi
$$

with $\phi=\operatorname{column}\left(\phi_{1}, \phi_{2}\right)$, and

$$
\tilde{\varepsilon}=\left(\varepsilon-\varepsilon_{0}\right) / 2 M \tilde{\lambda}^{2}
$$

Thus we reduce (3.21) to the system of two ordinary differential equations for radial functions, given by formula (3.23). Its solutions must be normalizable and vanish at $r=0$. For some types of potential $\varphi$ (and particular restrictions imposed on the coupling constants $e$ and $\lambda)$ this system is integrable and its solutions can be expressed via special functions. In the following section we consider an example of integrable equation (3.23) which corresponds to a neutral particle interacting anomalously with an external field. 


\section{RELATIVISTIC ANALOG OF PS PROBLEM}

Let us set $e=0$ in (2.14), (2.15) and choose the following particular realization for the potential $\varphi$ :

$$
\varphi=\omega \log (x)
$$

where $\omega$ is a constant. Then the related equations (3.17) and (3.23) are reduced to the following forms

$$
\varepsilon^{\prime} \chi=\left(\frac{p^{2}}{2 M}+\tilde{\lambda} \frac{\sigma_{\alpha} x_{\alpha}}{x^{2}}\right) \chi, \quad \varepsilon^{\prime}=\varepsilon-\varepsilon_{0}
$$

and

$$
H_{k} \phi \equiv\left(-\frac{\partial^{2}}{\partial r^{2}}+k\left(k-\sigma_{3}\right) \frac{1}{r^{2}}+\sigma_{1} \frac{1}{r}\right) \phi=\tilde{\varepsilon} \phi
$$

correspondingly, provided we set $\tilde{\lambda}=\omega \lambda$.

The electromagnetic field whose potential is defined by relations (2.5) and (4.25) has a transparent physical meaning. Namely, it is a superposition of the electric field $\mathbf{E}=\left(E_{1}, E_{2}, E_{3}\right)$ whose components are

$$
E_{1}=\omega \frac{x_{1}}{x^{2}}, \quad E_{2}=\omega \frac{x_{2}}{x^{2}}, \quad E_{3}=0
$$

and the magnetic field $\mathbf{B}=\left(B_{1}, B_{2}, B_{2}\right)$ with

$$
B_{1}=-\omega \frac{x_{2}}{x^{2}}, \quad B_{2}=\omega \frac{x_{1}}{x^{2}}, \quad B_{3}=0 .
$$

Such an electric field can be identified as the field of a charged infinite filament coinciding with the third coordinate axis. Let us designate the charge density of this filament by $\rho$ then the coupling constant $\omega$ should be equal to $2 \rho$. On the other hand, the magnetic field $\mathbf{B}$ is nothing but the field of a straight line constant current $j$ directed along the third coordinate axis provided the coupling constant $\omega$ be equal to $2 j$. Of course the related charge density and current should be equal between themselves, i.e.,

$$
j=\rho=\omega / 2 .
$$

Let us show that equation (4.26) is exactly solvable and find its solutions. The simplest way to prove integrability of (4.26) is to make the unitary transformation

$$
\chi \rightarrow \chi^{\prime}=U \chi, \varepsilon^{\prime}-\left(\frac{p^{2}}{2 M}+\tilde{\lambda} \frac{\sigma_{\alpha} x_{\alpha}}{x^{2}}\right) \rightarrow U\left(\varepsilon^{\prime}-\left(\frac{p^{2}}{2 M}+\tilde{\lambda} \frac{\sigma_{\alpha} x_{\alpha}}{x^{2}}\right)\right) U^{\dagger}
$$


where $U=\frac{1}{\sqrt{2}}\left(1-\mathrm{i} \sigma_{3}\right)$. As a result we reduce (4.26) to the following form:

$$
\varepsilon^{\prime} \chi=\left(\frac{p^{2}}{2 M}-2 \tilde{\lambda} \frac{S_{1} x_{2}-S_{2} x_{1}}{x^{2}}\right) \chi
$$

where $S_{1}=\frac{1}{2} \sigma_{1}$ and $S_{2}=\frac{1}{2} \sigma_{2}$ are spin matrices and in accordance with eqs.(2.3), (4.25) and (4.30) $\tilde{\lambda}=\lambda \omega=2 g \mu_{0} j$.

For a fixed $M$ and up to the value of the coupling constant equation (4.32) coincides with the Schrödinger equation for a neutral particle minimally interacting with the field generated by an infinite thin current filament (in our case the standard coupling constant is multiplied by factor 2). This equation was studied in numerous papers starting with [4] and continuing with [5]- 7] and many others. It has the following nice properties:

- equation (4.32) admits a hidden dynamical symmetry with respect to group $S O(3)$ for negative eigenvalues $\tilde{\varepsilon}$, group $S O(1,2)$ for $\tilde{\varepsilon}$ positive and group $E(2)$ for $\tilde{\varepsilon}=0$ [4];

- it possesses a hidden supersymmetry [5];

- using any of the above mentioned properties the equation can be integrated in closed form [4]-[6].

Since equation (4.26) is unitary equivalent to eq.(4.32) it succeeds the above mentioned properties. In particular, eigenvalues $\varepsilon^{\prime}$ are the same in both equations (4.26) and (4.32).

\section{RELATIVISTIC AND QUASI RELATIVISTIC ENERGY LEVELS}

In the next section we will present exact solutions of equation (4.26) for coupled states and define the related eigenvalues $\varepsilon^{\prime}$. In fact these eigenvalues are well known, and using directly results of paper [4] (or of the papers [5]-[8]) we can immediately write $\varepsilon^{\prime}$ in the following form:

$$
\varepsilon^{\prime}=-\frac{2 \tilde{\lambda}^{2} M}{N^{2}}
$$

where $N$ is a positive natural number.

Eigenvalues (5.33) are degenerated since they do not depend on eigenvalues $k$ of the angular momentum operator $J_{12}$. The degeneration factor is equal to $2 k+1$, and the quantum number $N$ can be represented as

$$
N=2(n+k)+1
$$


where $n$ is a natural number [4], [6].

Using (5.33) we already can find energy levels for the initial relativistic problem. Indeed, since $P_{0}=p_{0}+p_{3}$ and $P_{3}=\frac{1}{2}\left(p_{0}-p_{3}\right)$, it is possible to write analogous relations for eigenvalues $E$ of $p_{0}, \kappa$ of $p_{3}$ and $\varepsilon, M$ :

$$
\varepsilon=E+\kappa, \quad 2 M=E-\kappa .
$$

Then, using definitions (4.26) and (5.35) for $\varepsilon^{\prime}, E$ and $M$ we find from (5.33) the relativistic energy spectrum:

$$
E=\frac{m}{K+\tilde{\kappa}}+\kappa
$$

where

$$
K=\sqrt{1+\tilde{\kappa}^{2}+\frac{\tilde{\lambda}^{2}}{N^{2}}}, \quad \tilde{\kappa}=\frac{\kappa}{m}, \quad \tilde{\lambda}=2 \mu_{0} g j .
$$

We see that in spite of the fact that the neutron motion along the third coordinate axis is free, the third component of momentum $\kappa$ makes a rather non-trivial contribution into the values of energy levels (5.36). In accordance with (5.36) $E>\kappa$, and so the condition $M \neq 0$ is actually satisfied.

The most simple expression for energy levels corresponds to the particular value $\kappa=0$ :

$$
E=\frac{m}{\sqrt{1+\frac{\tilde{\lambda}^{2}}{N^{2}}}}
$$

which for small $\tilde{\lambda}$ becomes

$$
E=m\left(1-\frac{\tilde{\lambda}^{2}}{2 N^{2}}\right)+\cdots=m-\frac{2 m\left(g \mu_{0} j\right)^{2}}{N^{2}}+\cdots .
$$

Up to the rest energy term $m$ the approximate energy levels (5.39) are exactly the same as in the non-relativistic PS problem [4], [6]-[8]. In particular both the approximate and exact levels given by equations (5.36), (5.38) and (5.39) are degenerated with respect to eigenvalues $k$ of the third component of angular momentum which is a constant of motion for the considered system. Like in [4] this degeneration is caused by a hidden dynamical symmetry of the system.

Let both $\tilde{\lambda}$ and $\tilde{\kappa}$ are small. Expanding $E(\underline{5.39})$ in power series of $\tilde{\lambda}$ and $\tilde{\kappa}$ we obtain the quasi relativistic approximation for the energy levels:

$$
E \approx m+\frac{\kappa^{2}}{2 m}-\frac{\kappa^{4}}{8 m^{3}}-\frac{m \tilde{\lambda}_{\kappa}^{2}}{2 N^{2}}
$$

where

$$
\tilde{\lambda}_{\kappa}=(1-\tilde{\kappa}) \tilde{\lambda}
$$


The first tree terms in (5.40) represent respectively the rest energy, the kinetic energy of the free motion along the third coordinate axis and the relativistic correction to this energy. The last (dynamical) term in (5.40) is quite similar to the corresponding non-relativistic term (compare with (5.39) ), but includes the corrected coupling constant $\tilde{\lambda}_{\kappa}$ instead of $\tilde{\lambda}$.

Consider also the ultrarelativistic situation when large $\tilde{\kappa}$ is large but $\tilde{\lambda}$ is still small. Then the energy values (5.39) can be expanded as

$$
E=\sqrt{m^{2}+\kappa^{2}}-m \delta \frac{\tilde{\lambda}^{2}}{2 N^{2}}+\cdots
$$

where the dots denote the terms of order $\tilde{\lambda}^{4}$, and

$$
\delta=\left(2 \sqrt{1+\tilde{\kappa}^{2}}-2 \tilde{\kappa}-\frac{1}{\sqrt{1+\tilde{\kappa}^{2}}}\right) .
$$

Comparing (5.42) with (5.39) we recognize that the relativistic binding energy levels include the additional multiplier $\delta$ which considerable differs from 1 for ultrarelativistic $k$.

Notice that since the electromagnetic field defined by relations (2.5) and (2.6) has no components in $x_{3}$ direction, the motion of the particle in this direction is free. This motion can be quantized by imposing the periodic boundary condition. Then

$$
\kappa=\frac{2 \pi \tilde{N}}{L}, \tilde{N}=0, \pm 1, \pm 2, \cdots
$$

and energy levels (5.36) $-(\underline{5.42})$ be labeled by the pairs of quantum numbers $N$ and $\tilde{N}$.

\section{EXACT SOLUTIONS FOR BOUND STATES}

To find the solutions of equation (4.26) we use the fact that the Hamiltonian

$$
H_{k}=\left(-\frac{\partial^{2}}{\partial r^{2}}+k\left(k-\sigma_{3}\right) \frac{1}{r^{2}}+\sigma_{1} \frac{1}{r}\right)
$$

can be factorized as

$$
H_{k}=a_{k}^{+} a_{k}+C_{k}
$$

where

$$
a_{k}=\frac{\partial}{\partial r}+W_{k}, \quad a_{k}^{+}=-\frac{\partial}{\partial r}+W_{k}, \quad C_{k}=-\frac{1}{(2 k+1)^{2}}
$$

and $W$ is a matrix superpotential

$$
W_{k}=\frac{1}{2 r} \sigma_{3}-\frac{1}{2 k+1} \sigma_{1}-\frac{\left(k+\frac{1}{2}\right)}{r} .
$$


It can be verified by direct calculation that

$$
H_{k}^{+}=a_{k} a_{k}^{+}+C_{k}=-\frac{\partial^{2}}{\partial r^{2}}+(k+1)\left(k+1-\sigma_{3}\right) \frac{1}{r^{2}}+\sigma_{1} \frac{1}{r}
$$

i.e., the superpartner Hamiltonian $H_{k}^{+}$for $H_{k}$ is equal to $H_{k+1}$. Thus the eigenvalue problem (3.23) possesses a supersymmetry with shape invariance and so it can be solved using the standard technique of the supersymmetric quantum mechanics [21]. We will not reproduce the related routine calculations whose details can be found in [6], [7] but restrict ourselves to the presentation of the solutions of eq.(4.26).

The ground state solutions $\phi(0, k ; r)=$ column $\left(\phi_{1}(0, k ; r), \phi_{2}(0, k ; r)\right)$ are square integrable and normalizable solutions of equation $a_{k} \phi(0, k ; r)=0$. They can be expressed in the following form:

$$
\phi_{1}(0, k ; r)=r^{k+1} K_{1}\left(\frac{r}{2 k+1}\right), \quad \phi_{2}(0, k ; r)=-r^{k+1} K_{0}\left(\frac{r}{2 k+1}\right)
$$

where $K_{0}$ and $K_{1}$ are the modified Bessel functions. The corresponding eigenvalue $\tilde{\varepsilon}_{k}$ in (4.26) and $(\underline{3.23})$ is equal to $-\frac{1}{(2 k+1)^{2}}$.

Solutions corresponding to the first excited state, i.e., to $n=1$ are $\phi(1, k ; r)=a_{k}^{+} \phi(0, k+$ $1 ; r)$, or, being written componentwise,

$$
\begin{aligned}
\phi_{1}(1, k ; r) & =-\left(\frac{\partial}{\partial r}+\frac{k}{r}\right) \phi_{1}(0, k+1 ; r)-\frac{1}{(2 k+1)} \phi_{2}(0, k+1 ; r) \\
& =\frac{4(k+1)}{(2 k+1)(2 k+3)} r^{k+2} K_{0}\left(\frac{r}{2 k+3}\right)-(2 k+1) r^{k+1} K_{1}\left(\frac{r}{2 k+3}\right), \\
\phi_{2}(1, k ; r)= & -\left(\frac{\partial}{\partial r}+\frac{k+1}{r}\right) \phi_{2}(0, k+1 ; r)-\frac{1}{(2 k+1)} \phi_{1}(0, k+1 ; r) \\
& =(2 k+3) r^{k+1} K_{0}\left(\frac{r}{2 k+3}\right)-\frac{4(k+1)}{(2 k+1)(2 k+3)} r^{k+2} K_{1}\left(\frac{r}{2 k+3}\right) .
\end{aligned}
$$

The corresponding eigenvalue $\tilde{\varepsilon}_{k}$ is equal to $-\frac{1}{(2(k+1)+1)^{2}}=-\frac{1}{(2 k+3)^{2}}$.

Finally, solutions which correspond to an arbitrary value of the quantum number $n>0$ can be represented as

$$
\phi(n, k ; r)=a_{k}^{+} a_{k+1}^{+} \cdots a_{k+n-1}^{+} \phi(0, k+n ; r), \quad n=1,2, \cdots
$$


which gives rise to the recurrence relations

$$
\begin{aligned}
\phi_{1}(n, k ; r)= & -\frac{\partial}{\partial r} \phi_{1}(n-1, k+1 ; r)-\frac{k}{r} \phi_{1}(n-1, k+1 ; r) \\
& +\frac{1}{2 k+1} \phi_{2}(n-1, k+1 ; r), \\
\phi_{2}(n, k ; r)= & -\frac{\partial}{\partial r} \phi_{2}(n-1, k+1 ; r)-\frac{k+1}{r} \phi_{2}(n-1, k+1 ; r) \\
& +\frac{1}{2 k+1} \phi_{1}(n-1, k+1 ; r) .
\end{aligned}
$$

The related eigenvalue $\tilde{\varepsilon}_{k}$ is given by relations (5.33) and (5.34).

It is now possible to present exact solutions of the initial Dirac-Pauli equation defined by relations (2.1), (2.5) and (4.25). In accordance with the above such solutions are labeled by the main quantum number $N$ which can be expressed by equation (5.34), and by eigenvalues $\kappa$ and $k$ of the third component of momenta and total orbital momentum. Using equations (2.12), (2.13), (2.15), (3.20), (3.22) and (6.48)-(6.50) we find these solutions in the following form:

$$
\psi_{n, \kappa, k}=\frac{1}{\sqrt{2 \pi L r}} \exp \left(-\mathrm{i}\left(E x_{0}-\kappa x_{3}\right)\right)\left(\begin{array}{c}
\exp \left(i\left(k-\frac{1}{2}\right) \theta\right) \eta_{1}(n, k ; r) \\
\exp \left(i\left(k+\frac{1}{2}\right) \theta\right) \eta_{2}(n, k ; r) \\
\exp \left(i\left(k-\frac{1}{2}\right) \theta\right) \phi_{1}(n, k ; r) \\
\exp \left(i\left(k+\frac{1}{2}\right) \theta\right) \epsilon \phi_{2}(n, k ; r)
\end{array}\right) .
$$

Here $k$ and $n$ are nonnegative natural numbers,

$$
r=\frac{\sqrt{x_{1}^{2}+x_{2}^{2}}}{r_{0}}, \quad r_{0}=\frac{1}{M|\tilde{\lambda}|}=\frac{K+\tilde{\kappa}}{m|\tilde{\lambda}|}, \quad \epsilon=\frac{\tilde{\lambda}}{|\tilde{\lambda}|}
$$

where $\tilde{\kappa}=\kappa / m, E, K$ and $\kappa$ are given by equations (5.36), (5.37) and (5.44), $\phi_{1}(n, k ; r)$ and $\phi_{2}(n, k ; r)$ are functions defined by recurrence relations (6.48), (6.50),

$$
\begin{aligned}
& \eta_{1}(n, k ; r)=\tilde{\lambda}\left(\frac{\partial}{\partial r}+\frac{k}{r}\right) \epsilon \phi_{2}(n, k ; r)+(K+\tilde{\kappa}) \phi_{1}(n, k ; r), \\
& \eta_{2}(n, k ; r)=\tilde{\lambda}\left(\frac{\partial}{\partial r}-\frac{k}{r}\right) \phi_{1}(n, k ; r)+(K+\tilde{\kappa}) \epsilon \phi_{2}(n, k ; r) .
\end{aligned}
$$

Solutions (6.51) are normalizable and tend to zero with $r \rightarrow 0$. They are defined for nonnegative eigenvalues $k$ of the total angular momentum while solutions for $k$ negative can be obtained acting on (6.51) by the reflection operator $\hat{Q}=i \gamma_{0} \gamma_{2} \gamma_{3} R_{1}$ where $R$ is the reflection of the first spatial variable, i.e., $R_{1} \psi\left(x_{0}, x_{1}, x_{2}, x_{3}\right)=\psi\left(x_{0},-x_{1}, x_{2}, x_{3}\right)$ and $R_{1} \psi\left(x_{0}, r, \theta, x_{3}\right)=$ $\psi\left(x_{0}, r,-\theta, x_{3}\right)$. Using the Dirac matrices (2.8) we find the solutions with negative values of 
$k$ in the following form:

$$
\psi_{n, \kappa, k}=\frac{i}{\sqrt{2 \pi L r}} \exp \left(\mathrm{i}\left(E x_{0}-\kappa x_{3}\right)\right)\left(\begin{array}{c}
-\exp \left(-i\left(k+\frac{1}{2}\right) \theta\right) \eta_{2}(n, k ; r) \\
-\exp \left(i\left(\frac{1}{2}-k\right) \theta\right) \eta_{1}(n, k ; r) \\
\epsilon \exp \left(-i\left(k+\frac{1}{2}\right) \theta\right) \phi_{2}(n, k ; r) \\
-\exp \left(i\left(\frac{1}{2}-k\right) \theta\right) \phi_{1}(n, k ; r)
\end{array}\right)
$$

Let us present explicitly the components of solutions (6.51), (6.54) for $n=0$ and $n=1$. If $n=0$ then the related functions $\phi_{1}(n, k ; r)=\phi_{1}(0, k ; r)$ and $\phi_{2}(0, k ; r)$ are given by equation (6.48) while $\eta_{1}(0, k ; r)$ and $\eta_{2}(0, k ; r)$ have the following form:

$$
\begin{aligned}
& \eta_{1}(0, k ; r)=\Lambda_{k} \phi_{1}(0, k ; r)+\frac{\tilde{\lambda}(2 k+1)}{r} \phi_{2}(0, k ; r), \\
& \eta_{2}(0, k ; r)=\Lambda_{k} \phi_{2}(0, k ; r)
\end{aligned}
$$

where $\Lambda_{k}=\left(\frac{\tilde{\lambda}}{2 k+1}+K+\tilde{\kappa}\right)$. If $n=1$ the corresponding functions $\phi_{1}(1, k ; r)$ and $\phi_{2}(1, k ; r)$ are given by equations (6.49), and

$$
\begin{aligned}
\eta_{1}(1, k ; r) & =\Lambda_{k+1} \phi_{1}(1, k ; r)+\frac{\tilde{\lambda}(2 k+1)}{r} \phi_{2}(1, k ; r)-\frac{2 \tilde{\lambda}}{(2 k+3) r} \phi_{1}(0, k+1 ; r), \\
\eta_{2}(1, k ; r) & =\Lambda_{k+1} \phi_{2}(1, k ; r)+\frac{2 \tilde{\lambda}}{r} \phi_{1}(1, k ; r)+\frac{2 \tilde{\lambda}}{(2 k+3) r} \phi_{2}(0, k+1 ; r) \\
& +\frac{2(2 k+1) \tilde{\lambda}}{r^{2}} \phi_{1}(0, k+1 ; r)
\end{aligned}
$$

where $\Lambda_{k+1}=\left(\frac{\tilde{\lambda}}{2 k+3}+K+\tilde{\kappa}\right)$.

Functions $\eta_{a}(a=1,2)$ in (6.55) and (1.3) include the terms proportional to $\phi_{a}$ (the first terms in the r.h.s.). The remaining terms are small in comparison with $\phi_{a}$, which results in similarity of probability distributions for neutrons in our model to the distributions in the PS model, see Appendix.

\section{DISCUSSION}

In Sections 2 and 3 we study a class of Dirac-Pauli systems which can be effectively reduced to a set of Schrödinger-Pauli equations. The main inspiration for our research was to find an integrable relativistic formulation of the non-relativistic PS problem [4]. This goal cannot be achieved by a straightforward relativization of the PS problem since the Dirac-Pauli equation 
for a neutral particle interacting with the magnetic field generated by a filament current is not integrable.

In the present paper we succeed in obtaining an integrable relativistic model which in many aspects can be treated as an analogue of the PS model. To this end we introduce a superposition of magnetic and electric fields which is not equivalent to the field of straight current. Nevertheless, in the non-relativistic limit our model is reduced to the PS one. To justify this statement we return to equation (4.30) and note that in the CGS units it takes the following form:

$$
\rho=j / c
$$

where $c$ denotes the velocity of light.

In accordance with (7.57) the required charge density is small and tends to zero in the non-relativistic approximation when $c \rightarrow \infty$. Thus in the non-relativistic limit the external field which we consider reduces to the field used in the PS model. In addition, the energy spectrum (5.36) is reduced to the PS form, see equation (5.39). That is why we claim that the non-relativistic limit of the model defined by equations (2.1), (2.5) and (4.25) is exactly the PS model. This property can be directly proved using the Foldy-Wouthuysen transformation [22].

Let us discuss the obtained energy levels for coupled states in the quasi-relativistic approximation (5.40). Albeit the motion along the third Cartesian coordinate is free, the third momentum component $\kappa$ makes a contribution into the effective coupling constant $\tilde{\lambda}_{\kappa}$. The origin of this contribution is the anomalous interaction of neutron moving along the charged line with the magnetic field generated by this line. In the rest frame this motion is effectively changed by the current which flows in the line in the opposite direction, which is in perfect accordance with equation (5.41).

The contribution of $\kappa$ into the effective coupling constant $\tilde{\lambda}_{\kappa}$ (5.41) is small. Namely, it is proportional to the inverse speed of light. However, it affects the energy levels (5.40) much more than the relativistic correction to the kinetic energy $-\kappa^{4} / 8 m^{3}$ which is proportional to the squared inverse speed of light.

In the case of ultrarelativistic neutron motion along the charge and current carrying lines the contribution of the related momentum into the coupling energy becomes very essential. As it follows from (5.42) the distances between the energy levels can significantly differ from the non-relativistic ones since the multiplier $\delta$ (5.43) changes continuously from 0.121 (for $\tilde{\kappa} \rightarrow-1$ ) to 4.121 (for $\tilde{\kappa} \rightarrow 1$ ). 
In conclusion, we have presented a new exactly solvable problem for the Dirac-Pauli equation describing a neutral particle which interacts anomalously with a rather particular external field given by equations (4.28)-(4.30) having however a clear physical meaning. This type of anomalous interaction is the key to expand solutions of the problem via solutions of the $(1+2)$-dimensional Levi-Leblond equation invariant with respect to Galilei group. Moreover, the considered problem possesses a hidden symmetry and supersymmetry which cause the $(2 n+1)$-fold degeneration of the energy levels given by equations (5.36) and (5.34).

A natural question arises whether the considered relativistic problem with its symmetries is unique or there are other problems which can be effectively solved using reduction $\mathrm{SO}(1,3) \rightarrow \mathrm{HG}(1,2)$. In Sections 2 and 3 we study a certain class of such problems which can be effectively reduced to radial equation (3.23) which is exactly solvable when $e=0$ and $\varphi=\omega \log (x)$. We believe that there are other exactly solvable equations (3.23) and at least two of them can be immediately written down if we set $\varphi=\alpha / x$ and consider the alternative cases $e=0$ and $e \neq 0$. The related Dirac-Pauli equations (2.1), (2.5), (2.6) can be solved explicitly. We plane to study these and probably other integrable models in future.

\section{Acknowledgments}

One of co-authors (A.G.N.) acknowledges partial support by Miur project Cooperazione Internazionale AF 2007 with National Academy of Sciences OD Ukraine and Kwa-Zulu University Durban South Africa.

\section{Appendix: Coupling constants and probability distributions}

In the main text we consider an idealized model with the infinite thin current filament and charged line. To give an idea about its physical realizability let us discuss the probability density which corresponds to found solutions (6.51).

We formulated our problem for neutrons. However, the obtained results can be extended to other neutral particles which have non-trivial magnetic moments. As an example we consider here the sodium atoms in the ground state.

First we present in more transparent form the coupling constant $\tilde{\lambda}$ and scaling interval $r_{0}$ (6.52). Going from the Heaviside units to CGS ones we should make the following changes in 
equations (5.36) and (6.52):

$$
m \rightarrow m c^{2}, \quad \kappa \rightarrow c \kappa, \tilde{\kappa} \rightarrow \tilde{\kappa}^{\prime}=\frac{\kappa}{m c}, \tilde{\lambda} \rightarrow \frac{\tilde{\lambda}}{\hbar c}=\tilde{\lambda}^{\prime}, r_{0} \rightarrow \frac{2 C_{n}}{\left|\tilde{\lambda}^{\prime}\right|}\left(K+\frac{\kappa}{m c}\right)
$$

where $c$ is the velocity of light and $C_{n}$ is the Compton wave length for the neutron. The dimensionless constant $\tilde{\lambda}^{\prime}$ and the $r_{0}$ can be represented as

$$
\tilde{\lambda}^{\prime}=-\frac{g \alpha C_{n} N_{c} \hat{j}}{c}=7.633 \cdot 10^{-7} \hat{j}, \quad r_{0}=\frac{34.5 \AA}{\hat{j}}\left(K+\frac{\kappa}{m c}\right)
$$

where $\hat{j}=j / \mathrm{A}$ is the current measured in Amperes, $\alpha=\frac{e^{2}}{\hbar c}=\frac{1}{137}$ is the fine structure constant, $g=-3.82$ is the neutron Landé factor, $N_{c}=C / e=6.242 \cdot 10^{18}$ is the charge equal to one coulomb measured in elementary charges. Surely for realistic current values parameter $\tilde{\lambda}$ is small thus the expansions in power series of $\tilde{\lambda}$ made in Section $\mathrm{V}$ was well grounded.

We formulated our problem for neutrons. However, the obtained results can be extended to other neutral particles which have non-trivial magnetic moments. As an example we consider here the sodium atoms in the ground state. Then $|g| \rightarrow 5.4, m \rightarrow 23 m$, and the parameters (1.2) are transformed to the following ones:

$$
\tilde{\lambda}^{\prime}=4.68 \times 10^{-8} \hat{j}, \quad r_{0}=\frac{24.42 \AA}{\hat{j}}\left(K+\frac{\kappa}{m c}\right) .
$$

Consider now solutions (6.51) and evaluate the corresponding probability density:

$$
\bar{\psi}_{n, \kappa, k} \gamma_{0} r \psi_{n, \kappa, k}=C_{n, \kappa, k}^{2}\left(\phi_{1}^{2}+\phi_{2}^{2}+\tilde{\lambda}^{\prime}\left(K+\kappa^{\prime}\right) \epsilon \delta \frac{\partial\left(\phi_{1} \phi_{2}\right)}{\partial r}+\frac{\delta \tilde{\lambda}^{\prime 2}}{r} \frac{\partial\left(\phi_{2}^{2}-\phi_{1}^{2}\right)}{\partial r}\right)
$$

where $C_{n, \kappa, k}$ is a normalization constant, $\delta=\frac{1}{\left(K+\kappa^{\prime}\right)^{2}+1}$. In particular, for $n=0$,

$$
\bar{\psi}_{0, \kappa, k} \gamma_{0} r \psi_{0, \kappa, k}=C_{k}^{2}\left(\phi_{1}^{2}+\phi_{2}^{2}-\epsilon \delta_{1} \frac{\phi_{1} \phi_{2}}{r}+\delta_{2} \frac{\phi_{2}^{2}}{r^{2}}\right)
$$

where $\phi_{1}$ and $\phi_{2}$ are functions defined in (6.48) and

$$
\delta_{1}=2 \tilde{\lambda}^{\prime}\left((2 k+1)\left(K+\kappa^{\prime}\right)+\tilde{\lambda}^{\prime}\right) \delta, \quad \delta_{2}=\tilde{\lambda}^{\prime 2}(2 k+1)^{2} \delta
$$

Analysing (1.5) we conclude that the last two terms in the brackets are small. First they include the small multiplier $\tilde{\lambda}^{\prime}(\underline{1.2})$. Secondly, for $k>1 / 2$ functions $\frac{\phi_{1} \phi_{2}}{r}$ and $\frac{\phi_{2}^{2}}{r^{2}}$ are negligibly small in comparison with $\phi_{1}^{2}+\phi_{2}^{2}$. The same statement is correct for equation (1.4) which can be proven with using the identities

$$
\frac{\partial K_{0}(\lambda r)}{\partial r}=-\lambda K_{1}(\lambda r), \quad \frac{\partial K_{1}(\lambda r)}{\partial r}=-\lambda K_{0}(\lambda r)-\frac{1}{r} K_{1}(\lambda r) .
$$


Thus, practically without loss of accuracy, we can write

$$
\bar{\psi}_{n, \kappa, k} \gamma_{0} r \psi_{n, \kappa, k} \approx C_{n, \kappa, k}^{2}\left(\phi_{1}^{2}+\phi_{2}^{2}\right)
$$

and so the probability distribution calculated for our relativistic problem is virtually the same as the one obtained in [8] and [6] for the non-relativistic PS problem.

Thus the main statements presented in [8] and [6] concerning the possibility in principle to observe experimentally the neutrons and sodium atoms trapped by the current filament, can be generalized to our model, if we restrict ourselves to small $\tilde{\kappa}$. We will not repeat the reasonings given in the mentioned papers but remind that reasonable current values are $j \approx 50 \mathrm{~mA}$ for trapping neutrons and $j \approx 400 \mathrm{~mA}$ for trapping the sodium atoms in the ground state.

The principally new feature of the relativistic model is the essential dependence of the coupling energy and of the scaling parameter $r_{0}$ on the third component of momenta. In accordance with (5.42), (5.43) and (1.2), (1.3) for ultrarelativistic values of $\tilde{\kappa} \rightarrow 1$ the coupling energy obtains a non-unit multiplier which can increase up to $\sqrt{2}+1$ and $r_{0}$ has a multiplies which can increase up to 4 comparison with the case when $\tilde{\kappa}=0$ when these multipliers are the unit one. These facts can open new ways for experimental search for trapped neutral particles with non-trivial magnetic moments.

[1] V. G. Bagrov and D. M. Gitman Exact Solutions of Relativistic Wave Equations (Kluwer, 1990)

[2] L. Yang et al Rev. Sci. Instrum. 79031301 (2008).

[3] Y. Aharonov and A. Casher Phys. Rev. Lett. 53319 (2008).

[4] G. P. Pron'ko and Y. G. Stroganov Sov. Phys. JETP 451075 (1977).

[5] A. I. Voronin Phys. Rev. A 4329 (1991).

[6] L. V. Hau, G. A. Golovchenko and M. M. Burns Phys. Rev. Lett. 743138 (1995).

[7] R. de Lima Rodrigues, V. B. Bezerra and A. N. Vaidyac Phys. Lett. A 28745 (2001).

[8] R. Blúmel and K. Dietrich Phys. Lett. A 139236 (1989). R. Blúmel and K. Dietrich Phys. Rev. A 4322 (1991).

[9] G.V. Shishkin and V.M. Villalba J. Math. Phys. 345037 (1993).

[10] S. Bruce Phys. Scr. 64102 (2001).

[11] C. Berkdemir and C-F Cheng Phys. Scr. 70035003 (2009).

[12] Askold Duviryak SIGMA 4048 (2008). 
[13] J. Niederle and A. G. Nikitin J. Phys. A 390931 (2006).

[14] V. G. Bagrov and W. I. Fushchich and A. G. Nikitin Symmetries of Equations of Quantum Mechanics, (N.Y., Allerton Press Inc. 1994) .

[15] P.A. M. Dirac Rev. Mod. Phys. 21392 (1949).

[16] P. J. Redmond J. Math. Phys. 61163 (1965).

[17] J. Bergou and F. Ehlotzky Phys. Rev. A 272291 (1983).

[18] J. Patera, P. Winternitz and H. Zassenhaus J. Math. Phys. 161597 (1975).

[19] J. M. Lévy-Leblond Comm. Math. Phys. 6286 (1967).

[20] M. de Montigny, J. Niederle and A. G. Nikitin J. Phys. A 391 (2006).

[21] L. Gendenshtein JETP Lett. 38356 (1983).

[22] L.L Foldy and S.A. Wouthuysen Phys. Rev. 7829 (1950). 